\title{
One-Step Electrochemical Polymerization of Polyaniline Flexible Counter Electrode Doped by Graphene
}

\author{
Qi Qin, Fang He, and Wangxi Zhang \\ School of Materials and Chemical Engineering, Zhongyuan University of Technology, Zhengzhou 450007, China \\ Correspondence should be addressed to Qi Qin; qq@zut.edu.cn
}

Received 23 February 2016; Revised 14 June 2016; Accepted 29 June 2016

Academic Editor: Frédérique Ducroquet

Copyright ( 2016 Qi Qin et al. This is an open access article distributed under the Creative Commons Attribution License, which permits unrestricted use, distribution, and reproduction in any medium, provided the original work is properly cited.

\begin{abstract}
To improve the photoelectric property of polyaniline (PANI) counter electrode using for flexible dye-sensitized solar cell (DSSC), graphene (GN) was doped in PANI films covered on flexible conducting substrate by one-step electrochemical method, and then GN/PANI composites are characterized by scanning electron microscope (SEM), fourier transform infrared spectroscopy (FTIR), four probe instrument, and so on. The results show that PANI particles can be electrodeposited on the surface of GN sheets as the potential rising to $2.0 \mathrm{~V}$. This formed unique PANI-GN-PANI lamellar structure owing to the strong interaction of conjugated $\pi$ electron between GN and PANI results in the superior conductivity and catalytic performance of GN/PANI electrode. The maximum conversion efficiency of dye-sensitized solar cell with this counter electrode reaches $4.31 \%$, which is much higher than that of GN-free PANI counter electrode.
\end{abstract}

\section{Introduction}

DSSCs, a prominent member of the larger group of thin film photovoltaic devices, have been considered as a potential alternative to conventional Si-based solar cells for their low cost and high efficiency of energy conversion [1-3]. The conductivity and catalytic activity of counter electrodes (CEs), which catalyze the reduction of tri-iodide $\left(\mathrm{I}_{3}{ }^{-}\right)$ions to iodide $\left(\mathrm{I}^{-}\right)$ions and transfer electrons arriving from external circuit back to the redox electrolyte, affect the performance of photosensitive cell [4]. Pt CE, generally used for its small resistance and high catalytic performance, is extremely expensive and has the problem of reserves for large scale application [5]. So far, a plenty of tests have been tried to find an alternative noble material of Pt, such as carbon materials [6], tungsten carbide nanorods [7], alloy metals [8], and conductive polymer [9]. Furthermore, the ITO glass has the problem of high price and its fragility and shape limitation will limit the transportation of ITO glass based DSSCs. In recent years, the introduction of plastic films as substrates to fabricate a flexible counter electrode layer has drawn our attention [10-12].

PANI is one of the typical conducting polymers that attracts a great attention due to its ease of synthesis, nice environmental stability, interesting electroactivity, and simple acid/base doping/dedoping chemistry. Qin et al. [13] had fabricated PANI CEs based on flexible PET matrix, and the electrochemical properties of the prepared PANI and the photoelectric performance of DSSC assembled with PANI $\mathrm{CE}$ were also characterized. The result showed PANI CE based DSSC had low conversion efficiency because of the high internal resistance of PANI CE. Qin and Zhang [14] had reported that polyaniline was facilely synthesized on indium tin oxide (ITO) conducting polyethylene terephthalate substrates via electrochemical polymerization method, and the optimal efficiency of dye-sensitized solar cell (DSSC) with flexible PANI/ITO-PET counter electrode reached $0.86 \%$. Aiming to lower the resistance of PANI CE, a new improving PANI-CE composed with high conducting materials, such as various carbon materials, needs to be developed. GN has large theoretical surface area about $2620 \mathrm{~m}^{2} / \mathrm{g}$, can withstand large current densities [15], has extremely high charge (electrons and holes) mobility $\left(230,000 \mathrm{~cm}^{2} / \mathrm{Vs}\right)$, and has large amount of edge planes/defects [16]. Both GN and PANI have conjugated $\pi$ electrons, so PANI has a strong affinity for GN due to $\pi$ electron interactions, easily forming a uniform nanocomposite. Li et al. [17] had prepared polyaniline 
intercalated reduced graphene oxide CE on flexible Ti foil or PET substrate for DSSC application. Dinari et al. $[18,19]$ had prepared polyaniline-graphene quantum dots on FTO glass by in situ electrochemical polymerization and it showed good catalytic activity in promoting triiodide reduction. Herein, we consider synthesizing a flexible PANI-CE modified by GN on PET-ITO substrate via electropolymerization and characterize the performance of this GN/PANI CE based DSSC. The influence of GN nanosheets on the electric properties and photoelectro catalytic process of PANI CE has been also researched.

\section{Experimental}

2.1. Materials. Aniline (An, analytical grade from Sinopharm Chemical Reagent Co., Ltd.) was purified by distillation under reduced pressure prior to usage. GN (C>99.5 wt\%) nanosheets were bought from Nanjing Xianfeng nanomaterials technology Co., Ltd., ITO-PET films (Shenzhen Huanan Xiangcheng science and technology Co., Ltd. 10-30 $\Omega /$ sq) were used for PANI deposition. Analytical grade reagents, $\mathrm{H}_{2} \mathrm{SO}_{4}(98 \mathrm{wt} \%$ ) and ethanol (Sinopharm Chemical Reagent Co., Ltd.) were used without any pretreatment. $\mathrm{TiO}_{2}$ photo anodes, dye-N719, and iodine ion liquid electrolyte were the commercial product purchased from Tongna New materials Technology Company. All solutions were prepared from deionized water.

2.2. Fabrication of Flexible GN/PANI CE. The GN/PANI thin film was anodic deposited onto a PET-ITO substrate from a deposition bath consisting of $1 \mathrm{mg} / \mathrm{mL} \mathrm{GN}, 0.2 \mathrm{~mol} / \mathrm{L}$ aniline, and $0.5 \mathrm{~mol} / \mathrm{L} \mathrm{H}_{2} \mathrm{SO}_{4}$ in a three-electrode cell. The threeelectrode cell was constructed with a Pt sheet as CE and a saturated calomel electrode (SCE) as reference electrode. One ITO-PET sheet was used as a working electrode. The deposited area was $5 \mathrm{~mm} \times 5 \mathrm{~mm}$ with other area insulated by adhesive tapes. The potentiostatic polymerization was carried out using a computer-controlled electrochemical analyzer (CHI660C, Shanghai CH instruments, China). Prior to deposition, PET-ITO sheets were ultrasonically cleaned with ethanol for $10 \mathrm{~min}$. Then the potentiostatic polymerization of PANI flexible CE was carried out between $1.4 \mathrm{~V}$ and $2.2 \mathrm{~V}$ for $500 \mathrm{~s}$. Finally, resulting GN/PANI PET electrode was immersed in dilute sulphuric acid statically in order to expel aniline monomer and oligomer PANI from the polymeric film, followed by rinsing with deionized water for several times and dried in a vacuum at $60^{\circ} \mathrm{C}$ for $24 \mathrm{~h}$. By contract, a GN-free PANI flexible CE has also been prepared.

2.3. Assembling of DSSCs. Firstly $\mathrm{TiO}_{2}$ photoanode $(4 \mathrm{~mm} \times$ $4 \mathrm{~mm}$ ) coating on the ITO conducting glass plates by screen-printing was dipped overnight in a solution consisting of $0.5 \mathrm{mmol} / \mathrm{L}$ N719 dye (Ru(II)L2(NCS)2:2TBA, $\mathrm{L}=2,2^{\prime}$-bipyridyl-4,4'-dicarboxylic acid). Then, a dyesensitized solar cell was assembled by injecting a drop of liquid electrolyte with $\mathrm{I}_{2}(0.05 \mathrm{~mol} / \mathrm{L})$, LiI $(0.5 \mathrm{~mol} / \mathrm{L})$, 1methyl-3-propylimidazolium iodide $(0.4 \mathrm{~mol} / \mathrm{L})$, and 4 -tertbutylpyridine $(0.5 \mathrm{~mol} / \mathrm{L})$ in methoxy-propionitrile $(5 \mathrm{~mL})$

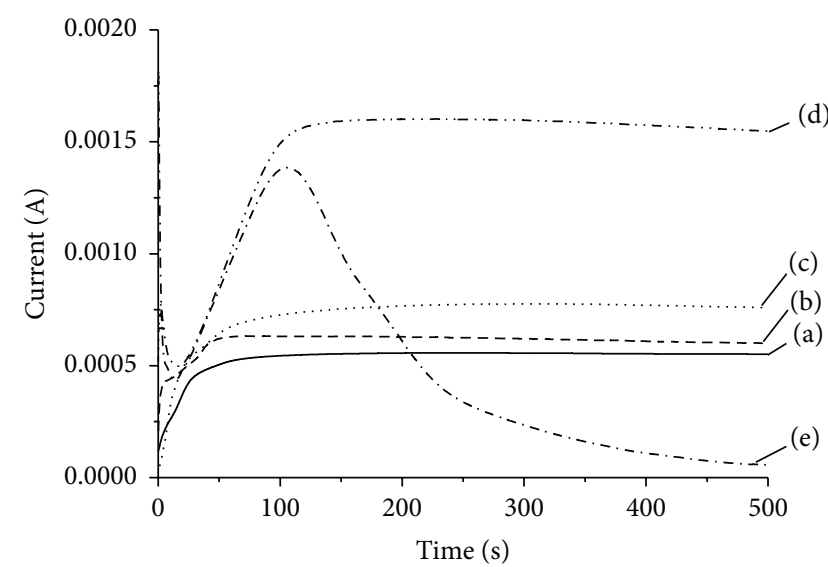

FIGURE 1: Current-time transients traced during GN/PANI composites at (a) $1.4 \mathrm{~V}$, (b) $1.6 \mathrm{~V}$, (c) $1.8 \mathrm{~V}$, (d) $2.0 \mathrm{~V}$, and (e) $2.2 \mathrm{~V}$.

into the aperture between the $\mathrm{TiO}_{2}$ electrode and the GN/PANI counter electrode. For the contrast test, the DSSCs with $\mathrm{TiO}_{2}$ photoanode and a pure-PANI CE were also fabricated.

2.4. Characterization of GN/PANI CE. We used a field-effect emission scanning electron microscopy (FE-SEM, JEOL JSM-7001F, and JPN) operated at $15 \mathrm{kV}$ to characterize the surface morphology of GN/PANI electrodes. Fourier transform infrared (FTIR) spectra were recorded in the range of $500-4000 \mathrm{~cm}^{-1}$ by a FTIR spectrometer (Nicolet iS10, USA). The conductivity of GN/PANI electrodes was measured by four-probe instrument (R9000, CHN). The thickness of samples was measured by micrometer caliper. The electrochemical impedance spectroscopy (EIS) was performed in a symmetric cell consisted of two identical counter electrodes with an electrochemical station (CHI660C, CH0020 Instrument, China) at the frequency range of $0.1-10^{5} \mathrm{~Hz}$. The magnitude of the alternative signal was $10 \mathrm{mV}$. Photocurrentvoltage $(J-V)$ characteristics of the DSSCs were obtained by a Keithley model 2400 digital source meter using an Oriel 91192 solar simulator equipped with AM 1.5 filter and intensity of $100 \mathrm{~mW} / \mathrm{cm}^{2}$. The effective cell area was $16 \mathrm{~mm}^{2}$.

\section{Results and Discussion}

3.1. Potentiostatic Polymerization. Figure 1 illustrates the current-time curves traced during the potentiostatic polymerization of GN/PANI at different constant potentials. It is shown that PANI doped with GN nanosheets can develop on the surface of ITO-PET substrate at $1.4 \mathrm{~V}$ by potentiostatic deposition, and there is little oxidation current below $1.4 \mathrm{~V}$. That is, GN/PANI film cannot be electrodeposited on PET film below $1.4 \mathrm{~V}$. The process of GN/PANI electrodeposited on conducting PET substrates always goes through two steps: the rapid growth period and the steady saturation period. During the initial stage, the current density increases sharply, and the initial rapid growth time is shortened within $50 \mathrm{~s}$ or so. It indicates that the nucleation rate upon $1.4 \mathrm{~V}$ is much 


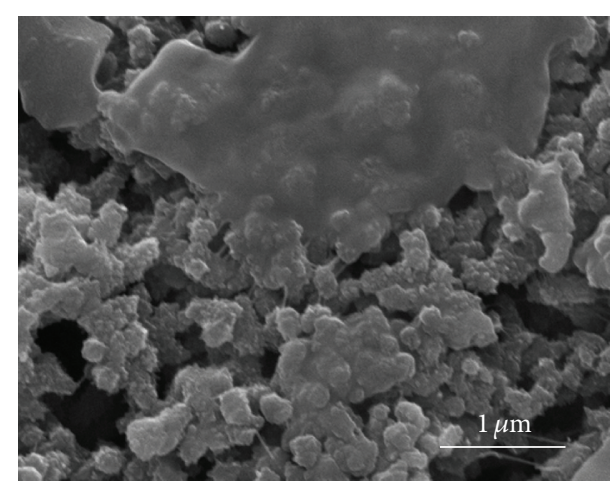

(a)

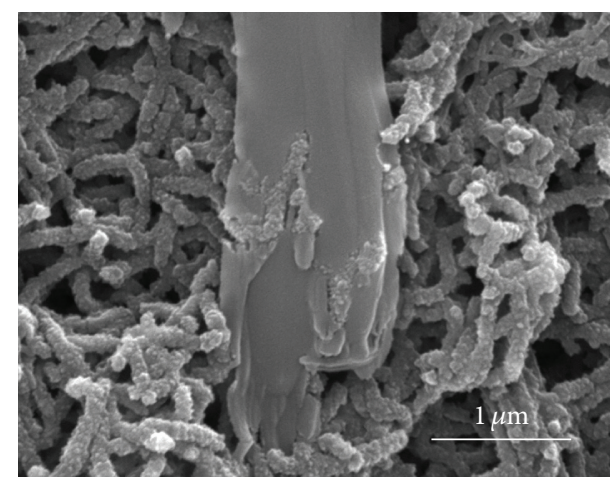

(c)

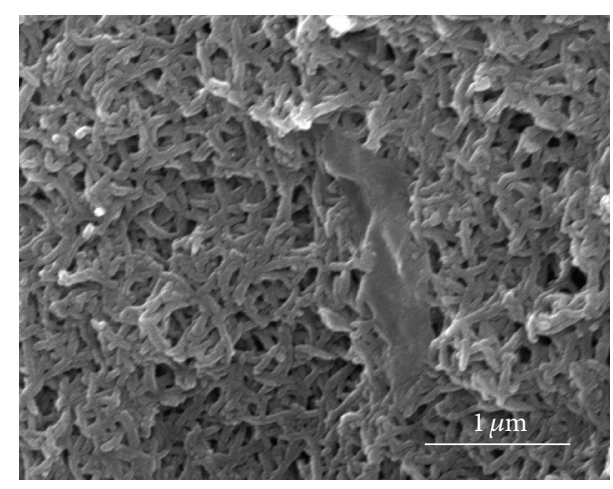

(b)

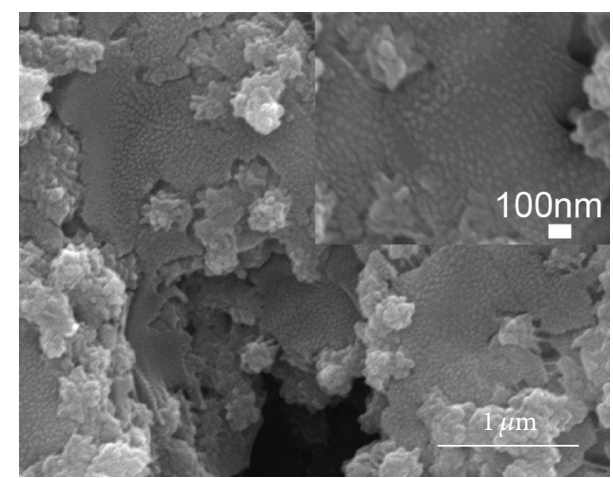

(d)

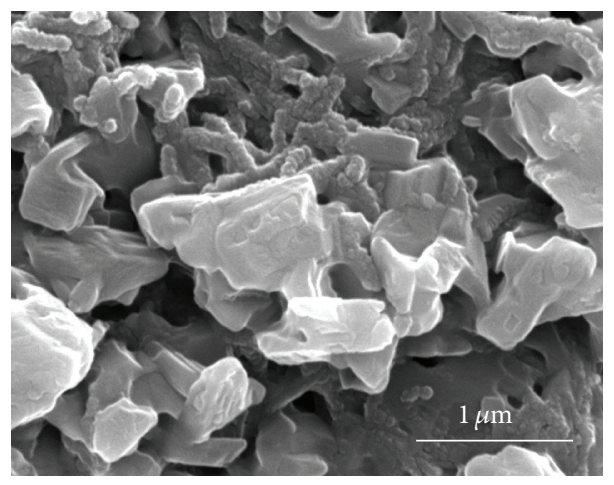

(e)

Figure 2: SEM photos of GN/PANI films at (a) $1.4 \mathrm{~V}$, (b) $1.6 \mathrm{~V}$, (c) $1.8 \mathrm{~V}$, (d) $2.0 \mathrm{~V}$, and (e) $2.2 \mathrm{~V}$.

rapid. This increase of anodic current depends on the oxidation of aniline to PANI during the growth process. Firstly, aniline cation radicals produced during the induction period adsorbed on the ITO-PET surface, then PANI chains grew from these primary nucleation centers, and soon the PET substrate was fully coated with a thin PANI film. Secondly, the secondary nucleation of PANI occurs already on the grown PANI film, and the PANI chains grew on the previous formed PANI film. It indicated that the growth rate of PANI would not be continually increasing but finally level off. After the induction period, the current density keeps steady for a long time, and the deposition process of PANI enters the saturated growth stage. Moreover, the current density gradually increases along with the rise of constant potential; that is, the growth rate of PANI electrodeposited at higher potential is much faster than that at low one. Especially, as the constant potential moved to more positive potential of $2.0 \mathrm{~V}$, the current density increased sharply threefold. This phenomenon might be attributed to the nucleation and growth of PANI take place on the surface of blended GN nanosheets when the constant potential reached $2.0 \mathrm{~V}$. This point can be also deduced from the micromorphology of GN/PANI film at $2.0 \mathrm{~V}$ by SEM (Figure 2(d)). However, the current density of PANI at $2.2 \mathrm{~V}$ declined followed the initial rapid growth period for $100 \mathrm{~s}$ or so. The decline of current intensity was related to the degradation of PANI film accompanying with the polymerization process of aniline. With the potential increasing (above $2.2 \mathrm{~V}$ ), the process of the peroxidation 
degradation was dominated, and this degradation phenomenon of PANI results in the damage of PANI film and the breakage of PANI molecular chain. Thus, the GN/PANI electrode cannot be well electropolymerized at $2.2 \mathrm{~V}$.

3.2. Morphological Properties. The morphologies of GN/ PANI films electrodeposited at different potentials were shown in Figure 2. The results showed that the few-layers GN nanosheets was doped into the PANI films. From Figure 2(a), it can be seen that some transparent, smooth, and paperlike GN nanosheets covered on the top of PANI particles or nanorods with the diameter of $120 \mathrm{~nm}$ or so, as PANI film electrodeposited on the surface of ITO-PET substrate at $1.4 \mathrm{~V}$. With the potential increasing to $1.6 \mathrm{~V}$, nanofiber PANI with the diameter of $40 \mathrm{~nm}$ and the length of more than $1 \mu \mathrm{m}$ can be formed. It is indicated that a preferential vertical growth of PANI particles occurs at higher potential, resulting in the formation of one-dimensional nanofiber PANI structure. Thus, very long length and low density of fiber PANI were observed on top of the compact basal layer, leading to a loosely porous structure. Doped GN sheets have similar effect on PANI prepared under $1.4 \mathrm{~V}$ and $1.6 \mathrm{~V}$. As the potential rising to $1.8 \mathrm{~V}$, PANI began to grow on the top of GN sheets. The one-dimensional nanofibrils PANI transformed to nanorods with the thicker diameter of $100 \mathrm{~nm}$. And on the top of partial GN nanosheets, some PANI nuclei particles with a diameter of $25 \mathrm{~nm}$ take place. Then it can be seen that many bulges appeared on the surface of GN layers with PANI electropolymerized under $2.0 \mathrm{~V}$. These are the similarspherical PANI particles grown on the entire GN layers. In addition, some big-sized PANI particles got together around the hybrid lamellar GN/PANI layers. When GN/PANI films are polymerized under $2.0 \mathrm{~V}$, the polymerization process of PANI occurs in two stages. Firstly, aniline monomers go in the gap of GN layers and are absorbed on the surface of smooth GN single layer. Secondly, aniline monomers electropolymerized on the GN layers, resulting in many special PANI spherical particles, which are quite different from the morphology of PANI prepared on ITO-PET substrates. This may be the result of the strong affinity between PANI nuclei and GN sheets due to both $\pi$ electron interactions, forming the uniform, compact coating of PANI nanoparticles. This PANI uniformly coated on the surface of the GN exhibited a hybrid lamellar structure, leading to excellent conductivity and absorbency.

Then with the polymerized potential increasing to $2.2 \mathrm{~V}$, the degradation of GN/PANI films took place. From Figure 2(e), it is shown that some aggregation of GN nanosheets and a few PANI nanorods piled up, and the PANI particles cannot grow on the surface of GN layers at overpotential. That is, the degradation and overoxidation of PANI were speeded by the ultrahigh potential. Thus, the growth of PANI nanoparticles or nanorods was destroyed. This result can be also demonstrated in Figure 1(e).

3.3. Fourier Transform Infrared Spectral Analysis. As shown in Figure 3, the broad peak in the frequency range of 3200$3500 \mathrm{~cm}^{-1}$ was attributed to the $\mathrm{N}-\mathrm{H}$ stretching vibrations of

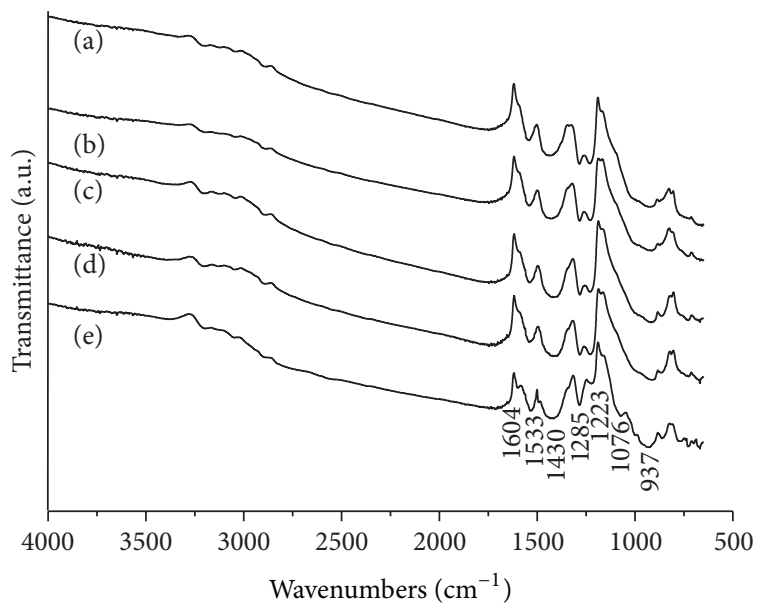

FIgURE 3: FTIR spectra of GN/PANI at (a) $1.4 \mathrm{~V}$, (b) $1.6 \mathrm{~V}$, (c) $1.8 \mathrm{~V}$, (d) $2.0 \mathrm{~V}$, and (e) $2.2 \mathrm{~V}$.

the emeraldine salt as for the PANI sample. The weak shoulders located at $2980-3050 \mathrm{~cm}^{-1}$ are ascribed to aromatic sp $\mathrm{C}-\mathrm{H}$ stretching. The absorption peaks at 1533 and $1430 \mathrm{~cm}^{-1}$ are corresponding to the $\mathrm{C}=\mathrm{C}$ stretching deformation of the quinoid ring and benzenoid rings, respectively, in the emeraldine salt. The peaks at 1285 and $1223 \mathrm{~cm}^{-1}$ correspond to $\mathrm{C}-\mathrm{N}$ stretching of the secondary aromatic amine and $\mathrm{C}=\mathrm{N}$ stretching, respectively. The peaks located at 937 and $864 \mathrm{~cm}^{-1}$ correspond to the aromatic $\mathrm{C}-\mathrm{H}$ bending in the plane and out of the plane for the 1,4-disubstituted aromatic ring. The result shows the PANI can be successfully synthesized on the surface of ITO-PET by electropolymerization.

For GN/PANI electrode at 2.2 V (Figure 3(e)), the characteristic band at $1223 \mathrm{~cm}^{-1}$, which was related to $\mathrm{C}=\mathrm{N}$ stretching modes, turned to be more weakening. The reason is the change of PANI structure caused by the overoxidation phenomenon, whereas it is clear that the characteristic peak of GN presents the carbon rings stretching deformation at $1076 \mathrm{~cm}^{-1}$ increased due to the degradation of PANI, and the characteristic peaks of the aggregated GN sheets appeared more obviously.

3.4. Conductivity Measurement. The conductivity of GN/ PANI polymerized at different potential is measured by four-point-probe instrument. Table 1 presents the thickness and the conductivity of pure PANI and GN/PANI layers at different potential. It can be found that the film thickness of GN/PANI increases with the polymerization potential increasing, while the conductivity decreases. Except the GN/PANI film at $2.0 \mathrm{~V}$, the conductivity is increasing to $0.375 \mathrm{~S} / \mathrm{cm}$. It may be contributed to the PANI particles growth on the surface of GN sheets with lower resistance at high potential of $2.0 \mathrm{~V}$, resulting in the improvement of conductivity of PANI electrode. In addition, the thickness of GN/PANI film was increased little and poorer conductivity of GN/PANI film electrodeposited at $2.2 \mathrm{~V}$ is caused by the degradation and overoxidation of PANI. As the polymerization potential reached too high values, the degradation 
TABLE 1: The thickness and conductivity of GN/PANI.

\begin{tabular}{lccc}
\hline Sample & $\begin{array}{c}\text { Resistance } \\
\Omega\end{array}$ & $\begin{array}{c}\text { Thickness } \\
\mu \mathrm{m}\end{array}$ & $\begin{array}{c}\text { Conductivity } \\
\text { S/cm }\end{array}$ \\
\hline $1.4 \mathrm{~V}$ & 520 & 16.2 & 0.262 \\
$1.6 \mathrm{~V}$ & 440 & 21.0 & 0.239 \\
$1.8 \mathrm{~V}$ & 340 & 30.2 & 0.215 \\
$2.0 \mathrm{~V}$ & 120 & 49.0 & 0.375 \\
$2.2 \mathrm{~V}$ & 380 & 50.0 & 0.116 \\
PANI & 1730 & 36.7 & 0.035 \\
\hline
\end{tabular}

of GN/PANI took place, leading to the breakage of molecular chain and the damage of PANI film. Meanwhile, the GN nanosheets without PANI particles covered aggregated seriously; thus the conductivity of GN/PANI at $2.2 \mathrm{~V}$ was reduced much. Thus, the best conductivity of GN/PANI deposited on conducting PET substrate reached $0.375 \mathrm{~S} / \mathrm{cm}$ when the polymerization potential was $2.0 \mathrm{~V}$. To compare, the conductivity of pure PANI film was measured. It can be seen that the resistance of pure PANI was higher than PANI with doped GN, due to $\pi$ electron conjugative effect between GN and PANI, speeding up the electron transfer rate. GN doped in PANI can reduce the resistance of film, and the conductivity was increasing about one order of magnitude.

3.5. Electrochemical Impedance Spectroscopy. In order to characterize the electrochemical behavior of the GN/PANI composite film, EIS analysis was carried out with the symmetric cell consisting of two identical GN/PANI electrodes prepared at $2.0 \mathrm{~V}$. The interelectrode space was filled with same content of liquid electrolyte. The Nyquist plots of symmetric cells for these various counter electrodes are shown in Figure 4. The equivalent circuit model of the EIS spectra is also drawn in Figure 4. $R_{s}$ describes mainly the ohmic serial resistance of the two identical electrodes. $R_{\mathrm{ct}}$ measures the charge transfer resistance of electrolytes.

From Figure 4, it can be obviously observed that the resistance of PANI doped by GN markedly decreases, compared to that of pure PANI, which illustrates that the addition of GN enhances the conductivity of the polymer significantly. The resistance of PANI-GN composite electrode is reduced with the content of GN increasing. On the other hand, the conductivity measurement also shows that the addition of GN benefits charge transport in the polymer conductor, which is consistent with the results obtained by EIS spectra simultaneously.

The Nyquist plots (EIS spectra) of both polymers with and without GN exhibit the semicircular characteristics in the measured range of frequency without linear behavior in the low-frequency range, which indicates that the controlling step in the electrode reaction is charge transfer, but not ionic diffusion. It is indicated that the conductivity of polymers in the presence or absence of GN comes from charge transport but not ionic conductivity. Compared to the pure PANI, GN/PANI composite electrode is believed to show the faster charge transfer owing to the excellence conductivity of GN

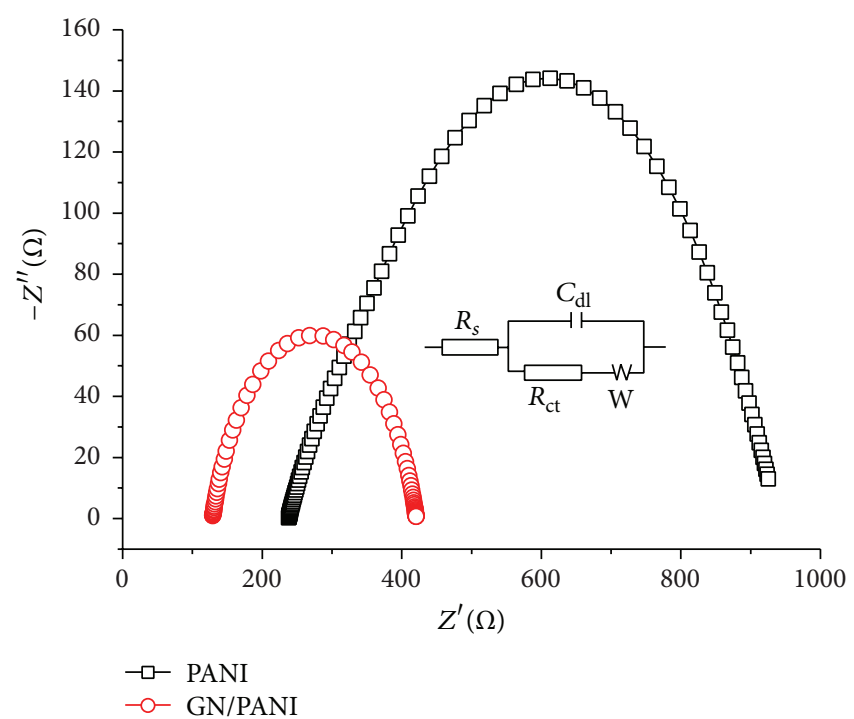

FIGURE 4: Nyquist plots of electrochemical cells consisting of various electrodes (equivalent circuit model of the EIS spectra. W: Warburg diffusion impedance; $R_{\mathrm{ct}}$ : charge transfer resistance; $C_{\mathrm{dl}}$ : capacitance of electric double layer; $R_{s}$ : ohmic serial resistance).

nanosheets and $\pi$ electron interactions between GN and PANI. The $R_{s}$ values of GN/PANI are more than $130 \Omega$, indicating that the significantly high sheet resistance of two electrodes with ITO conducting PET substrate leads to a large value of $R_{s}$. $R_{\mathrm{ct}}$ is obviously reduced with the added amount of GN; it can be deduced that the GN/PANI composite electrode had lower charge transfer resistance for liquid electrolyte. Owing to the excellent conductivity and absorbability of GN nanosheets, the catalytic rate of GN/PANI for liquid electrolyte is increasing, and the charge transfer rate of GN/PANI composite is faster than that of pure PANI.

3.6. IV Measurement. The photocurrent-photovoltage curves of DSSCs with different counter electrode are shown in Figure 5. And the photovoltaic characteristics of the DSSCs are also summarized in Table 2. The photoelectric conversion efficiency can be calculated by the following equation:

$$
\eta(\%)=\frac{V_{\mathrm{oc}} \times J_{\mathrm{sc}} \times \mathrm{FF}}{P_{\mathrm{in}}} \times 100,
$$

where $J_{\mathrm{sc}}$ is short-circuit photocurrent density $\left(\mathrm{mA} / \mathrm{cm}^{2}\right), V_{\mathrm{oc}}$ is open-circuit voltage $(\mathrm{mV}), \mathrm{FF}$ is fill factor, and $P_{\text {in }}$ is the incident power. The photo-to-current conversion efficiency of GN/PANI CE based DSSCs shows a gradual decrease from $1.4 \mathrm{~V}$ to $2.2 \mathrm{~V}$, but there is a prominent enhancement of conversion efficiency with GN/PANI CE at $2.0 \mathrm{~V}$ (4.31\%), which is much higher than that of DSSC based on the GN-free PANI CE (1.75\%). Of course, a great increase of the photocurrent of DSSC with GN/PANI CE at $2.0 \mathrm{~V}$ is attributed to the higher electron exchange efficiency and higher specific surface area of doped GN sheets. It was found that the GN nanosheets had higher specific surface area, leading to the much more absorption of liquid electrolyte, 


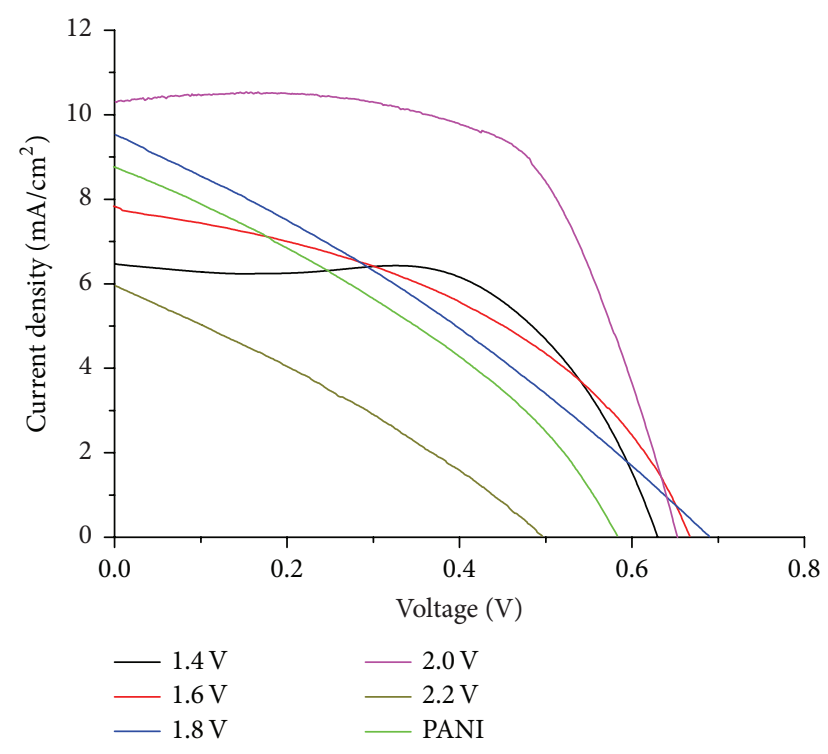

FIGURE 5: IV curves of DSSCs with different counter electrode.

TABLE 2: Photovoltaic parameter of DSSCs with different counter electrodes.

\begin{tabular}{lcccc}
\hline CEs & $\begin{array}{c}V_{\text {oc }} \\
\mathrm{V}\end{array}$ & $\begin{array}{c}J_{\text {sc }} \\
\mathrm{mA} / \mathrm{cm}^{2}\end{array}$ & FF & $\begin{array}{c}\eta \\
\%\end{array}$ \\
\hline GN/PANI, 1.4 V & 0.63 & 6.5 & 0.62 & 2.52 \\
GN/PANI, 1.6 V & 0.67 & 7.8 & 0.43 & 2.26 \\
GN/PANI, 1.8 V & 0.69 & 9.5 & 0.30 & 1.99 \\
GN/PANI, 2.0 V & 0.65 & 10.3 & 0.64 & 4.31 \\
GN/PANI, 2.2 V & 0.50 & 5.9 & 0.29 & 0.88 \\
PANI & 0.58 & 8.8 & 0.34 & 1.75 \\
\hline
\end{tabular}

which is benefitted for charge transfer between the electrolyte and counter electrode. PANI films cannot be formed on the GN sheets at low potential of polymerization, and the relatively poor conductivity of GN/PANI CE resulted in the higher charge transfer resistance of cells and the drop of conversion efficiency. As the potential rises to $2.0 \mathrm{~V}$, newly PANI was polymerized on the surface of GN layer and formed many nanoparticle PANI layers with high specific area. This hybrid GN/PANI lamellar structure gives rise to the excellent conductivity and absorbency; as a result, the short-circuit photocurrent and fill factor of this DSSC increased obviously, leading to the rise of the photoelectric conversion efficiency of cells. In conclusion, the photovoltaic performance of PANI CEs can be extremely improved by the modification of GN, owing to the fierce binding force of PANI with GN, and the excellent conducting property of the composites.

\section{Conclusions}

In this study, unique GN/PANI hybrid composite electrodes were obtained by a facile potentiostatic deposition in an aqueous electrolyte containing GN nanosheets and employed for DSSCs. It exhibits the uniform PANI nanofibrils or particles coated on the surface of $\mathrm{GN}$ at $2.0 \mathrm{~V}$, which had a lower charge transfer resistance and a superior phototo-current conversion efficiency of $4.31 \%$, in comparison with a pure PANI electrode, due to the synergistic effect between GN and PANI. The flexible GN/PANI electrode can be a promising alternative as a counter electrode applied in flexible DSSCs for its low cost, high conducting property, and electrocatalytic activity.

\section{Competing Interests}

The authors declare that the received grants, scholarships, or funding mentioned in Acknowledgments does not lead to any conflict of interests regarding the publication of this manuscript.

\section{Acknowledgments}

This work was supported by Henan Science and Technology Department (no. ZR2010BM038).

\section{References}

[1] X. Li, H. Lin, J. B. Li, X. Li, B. Cui, and L. Zhang, "A numerical simulation and impedance study of the electron transport and recombination in binder-free $\mathrm{TiO}_{2}$ film for flexible dyesensitized solar cells," Journal of Physical Chemistry C, vol. 112, no. 35, pp. 13744-13753, 2008.

[2] D. Zhang, T. Yoshida, T. Oekermann, K. Furuta, and H. Minoura, "Room-temperature synthesis of porous nanoparticulate $\mathrm{TiO}_{2}$ films for flexible dye-sensitized solar cells," Advanced Functional Materials, vol. 16, no. 9, pp. 1228-1234, 2006.

[3] M. Toivola, T. Peltola, K. Miettunen, J. Halme, and P. Lund, "Thin film nano solar cells-from device optimization to upscaling," Journal of Nanoscience and Nanotechnology, vol. 10, no. 2, pp. 1078-1084, 2010.

[4] M. Ikegami, K. Miyoshi, T. Miyasaka et al., "Platinum/titanium bilayer deposited on polymer film as efficient counter electrodes for plastic dye-sensitized solar cells," Applied Physics Letters, vol. 90, no. 15 , p. $153122,2007$.

[5] L. Chen, W. Tan, J. Zhang, X. Zhou, X. Zhang, and Y. Lin, "Fabrication of high performance Pt counter electrodes on conductive plastic substrate for flexible dye-sensitized solar cells," Electrochimica Acta, vol. 55, no. 11, pp. 3721-3726, 2010.

[6] C.-M. Chen, C.-H. Chen, and T.-C. Wei, "Chemical deposition of platinum on metallic sheets as counterelectrodes for dyesensitized solar cells," Electrochimica Acta, vol. 55, no. 5, pp. 1687-1695, 2010.

[7] P. Vijayakumar, M. Senthil Pandian, S. P. Lim et al., "Facile synthesis of tungsten carbide nanorods and its application as counter electrode in dye sensitized solar cells," Materials Science in Semiconductor Processing, vol. 39, pp. 292-299, 2015.

[8] B. L. He, Q. W. Tang, H. H. Zhang, and L. Yu, "Counter electrode electrocatalysts from binary Pd-Co alloy nanoparticles for dyesensitized solar cells," Solar Energy, vol. 124, pp. 68-75, 2016.

[9] M. Izu and T. Ellison, "Roll-to-roll manufacturing of amorphous silicon alloy solar cells with in situ cell performance diagnostics," Solar Energy Materials and Solar Cells, vol. 78, no. 1-4, pp. 613-626, 2003. 
[10] J. Hou, Z. Liu, and P. Zhang, "A new method for fabrication of graphene/polyaniline nanocomplex modified microbial fuel cell anodes," Journal of Power Sources, vol. 224, pp. 139-144, 2013.

[11] J.-J. Chen, W.-W. Li, X.-L. Li, and H.-Q. Yu, "Improving biogas separation and methane storage with multilayer graphene nanostructure via layer spacing optimization and lithium doping: a molecular simulation investigation," Environmental Science \& Technology, vol. 46, no. 18, pp. 10341-10348, 2012.

[12] Q.-B. Meng, K. Takahashi, X.-T. Zhang et al., "Fabrication of an efficient solid-state dye-sensitized solar cell," Langmuir, vol. 19, no. 9, pp. 3572-3574, 2003.

[13] Q. Qin, J. Tao, and Y. Yang, "Preparation and characterization of polyaniline film on stainless steel by electrochemical polymerization as a counter electrode of DSSC," Synthetic Metals, vol. 160, no. 11-12, pp. 1167-1172, 2010.

[14] Q. Qin and R. Zhang, "A novel conical structure of polyaniline nanotubes synthesized on ITO-PET conducting substrate by electrochemical method," Electrochimica Acta, vol. 89, pp. 726731, 2013.

[15] A. K. Geim and K. S. Novoselov, "The rise of graphene," Nature Materials, vol. 6, no. 3, pp. 183-191, 2007.

[16] K. S. Novoselov, A. K. Geim, S. V. Morozov et al., "Twodimensional gas of massless Dirac fermions in graphene," Nature, vol. 438, no. 7065, pp. 197-200, 2005.

[17] R. Li, Q. W. Tang, L. M. Yu, X. F. Yan, Z. M. Zhang, and P. Z. Yang, "Counter electrodes from conducting polymer intercalated graphene for dye-sensitized solar cells," Journal of Power Sources, vol. 309, pp. 231-237, 2016.

[18] M. Dinari, M. M. Momeni, and M. Goudarzirad, "Nanocomposite films of polyaniline/graphene quantum dots and its supercapacitor properties," Surface Engineering, vol. 32, no. 7, pp. 535-540, 2016.

[19] M. Dinari, M. M. Momeni, and M. Goudarzirad, "Dyesensitized solar cells based on nanocomposite of polyaniline/ graphene quantum dots," Journal of Materials Science, vol. 51, no. 6, pp. 2964-2971, 2016. 

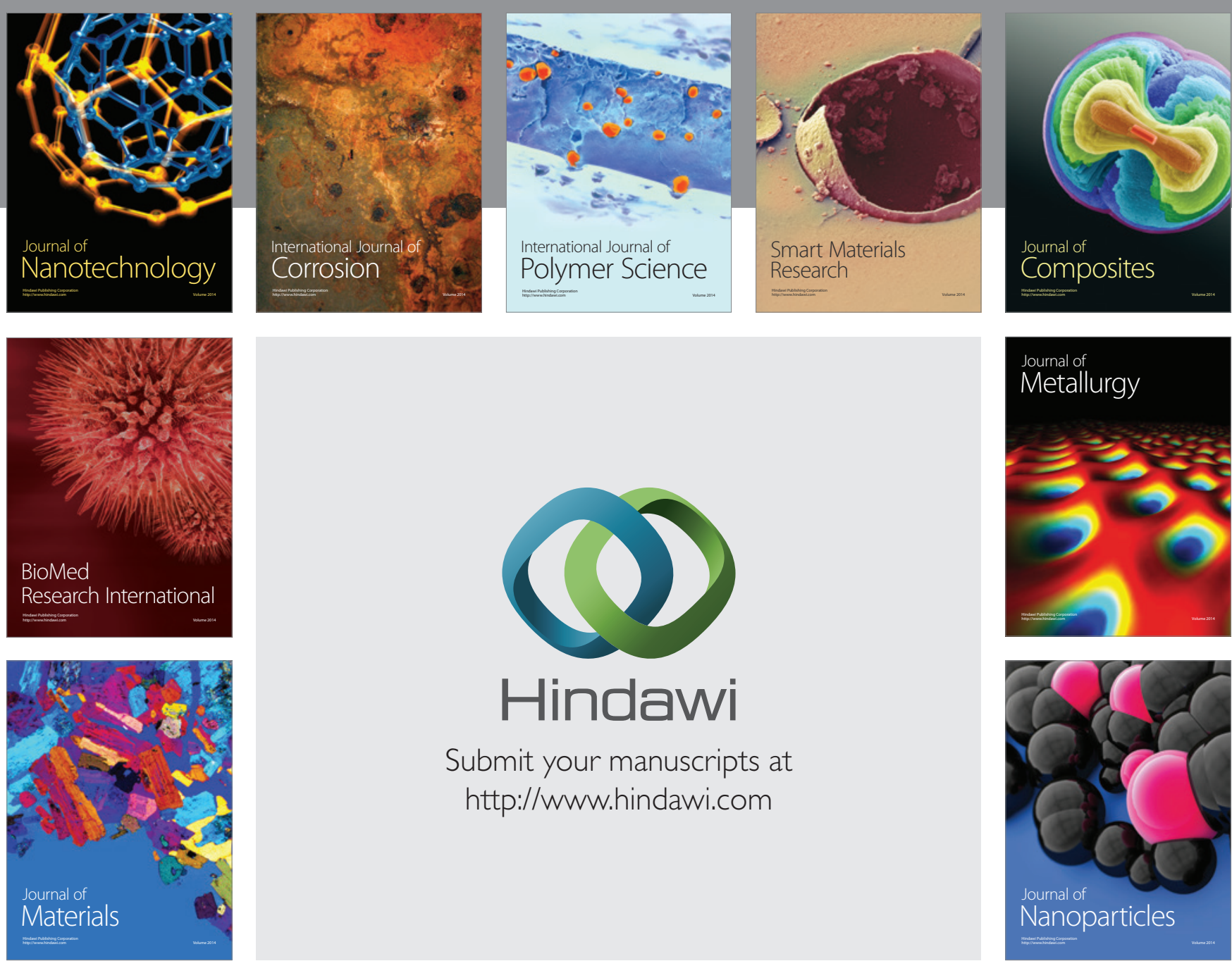

\section{Hindawi}

Submit your manuscripts at

http://www.hindawi.com

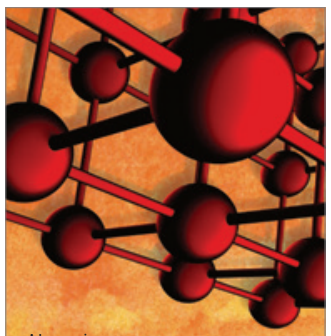

Materials Science and Engineering
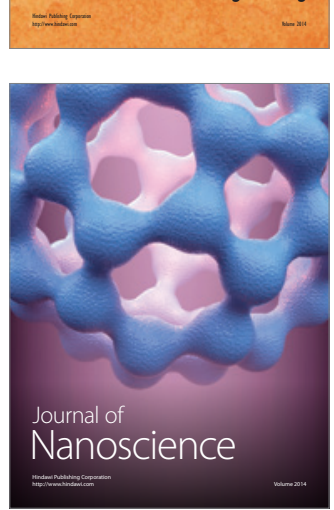
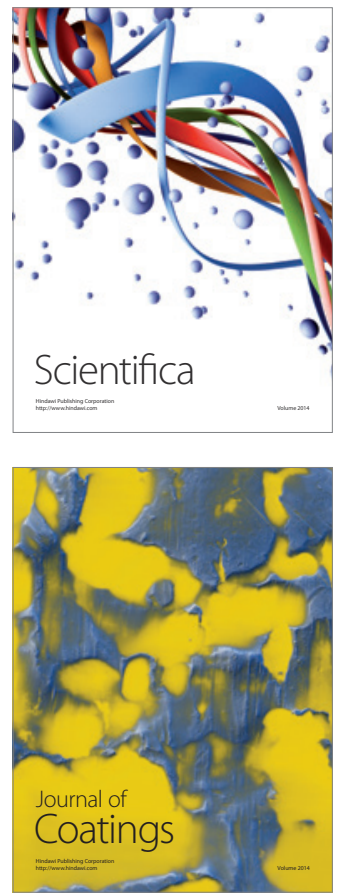
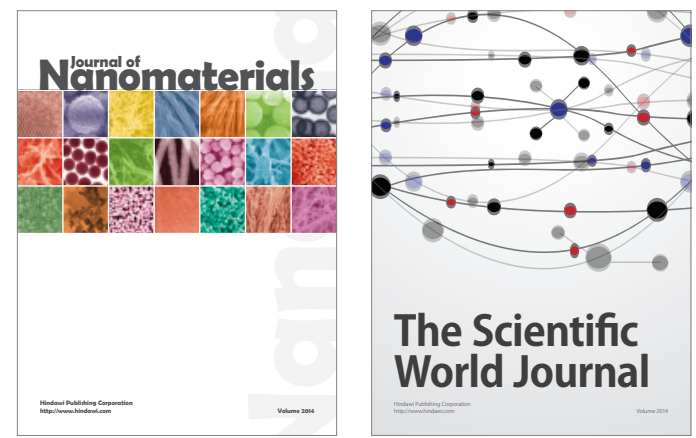

The Scientific World Journal
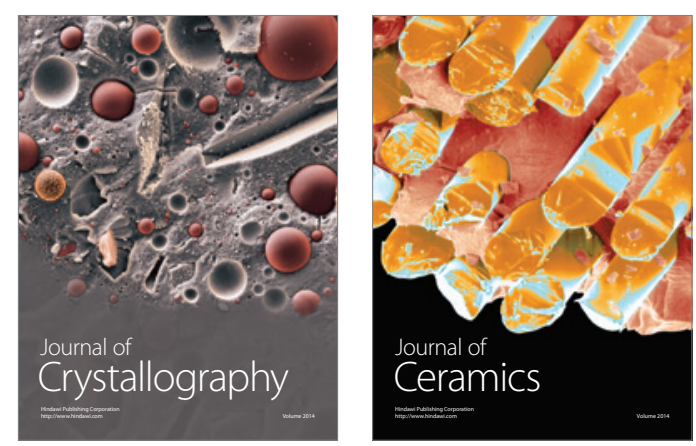
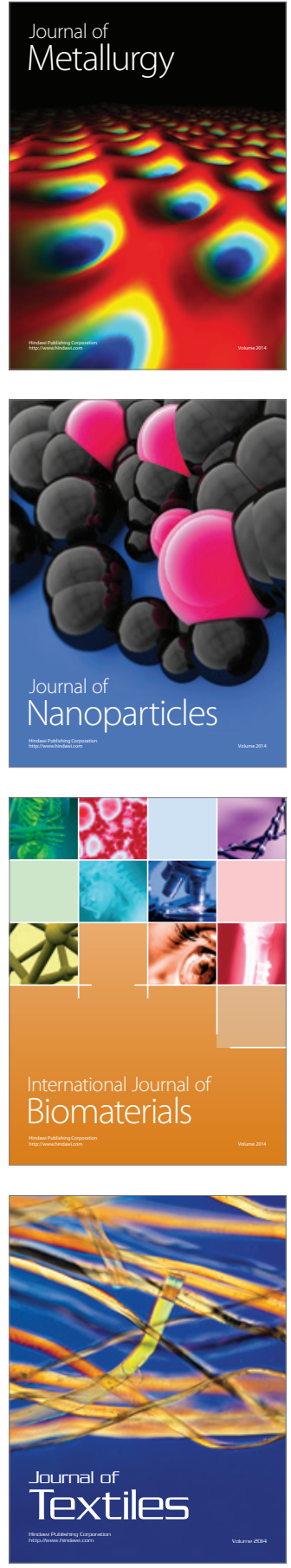